\title{
Impacts of COVID-19 Pandemic and Quality of Life in Women Immediately after Childbirth: Observational Study
}

\author{
Revathi. S', Sukriti Rijal², Vijaya Krishna Kumar ${ }^{3}$ \\ ${ }^{1}$ Associate Professor, ${ }^{2}$ BPT Intern, ${ }^{3}$ Principal, \\ Dr. B.R. Ambedkar Medical College \& Hospital, Department of Physiotherapy, \\ Rajiv Gandhi University of Health Sciences. Bengaluru, Karnataka \\ Corresponding Author: Revathi. S
}

DOI: https://doi.org/10.52403/ijhsr.20220310

\begin{abstract}
Background: The pandemic has burst due to novel corona virus which is ruled out from Wuhan city, China. Therefore, it provided a chance to investigate about impacts of Covid-19 pandemic in women immediately after childbirth \& quality of life that has settled to many people lifestyle especially in obstetrics.
\end{abstract}

Aim: To investigate about impacts of Covid-19 pandemic and quality of life in women immediately after childbirth.

Study Design: Observational study

Method: 100 sample sizes were taken between the age of 18-39 years based on inclusion and exclusion criteria. This survey is collected in standard demographic data and included questions that addresses topics such as quality of life, Covid-19 exposures, worries \& lifestyle changes by using WHOQOL-BREF scale.

Result: The aim of the study was to find out the impacts of covid-19 pandemic on quality of life in women immediately after child birth. The significance level was set to $\mathrm{p}<0.05$. There was a significant impact of Covid-19 pandemic on quality of life in women immediately after childbirth.

Conclusion: Centered on the result of the current study it is concluded that there was a significant impact of COVID-19 pandemic on quality of life in women immediately after childbirth because all the test shows $\mathrm{p}<0.05$.

Keywords: Covid-19, Childbirth, Quality of Life, Pandemic.

\section{INTRODUCTION}

The COVID-19 is a contagious disease that was recognize in Wuhan China, in Dec 2019. Many lives and health of people are affected worldwide with millions of cases and thousands of deaths due to COVID-19. ${ }^{[1]}$ The difficulties or exceptions facing in women immediately after childbirth have been studied in this time due to severity of COVID19 in lives and wellbeing of people with more risk of adverse neonatal \& pregnancy outcomes. ${ }^{[2]}$
Due to COVID-19 pandemic there has been notice that the level of worry, sleeping disorders, sorrow, worry and lack of physical activity increased in the largely population. ${ }^{[3]}$ In the starting research case and studies shows increased neonatal mortality, maternal mortality and morbidity and adverse pregnancy outcomes due to COVID-19. ${ }^{[3,4]}$ Also risk in mental health such as stress, unhappiness, loneliness, posttraumatic stress syndrome and isolation. ${ }^{[2,5,6]}$

Earlier studies have examined worries related to the well-being of the 
Revathi. S et.al. Impacts of COVID-19 pandemic and quality of life in women immediately after childbirth: observational study.

fetus, psychological fatigue and stressful life events that are associated with preterm birth and low birth weight ${ }^{[4,6]}$

Because of the restrictions in support person on attending prenatal appointment, the loss of in person labor and courses of delivery may lead women to not well informed \& not supported by their providers. ${ }^{[1,7]}$

In this study it is focused on how the COVID-19 pandemic is affecting quality of life among women immediately after childbirth based on WHOQOL-BRIEF SCALE which consist of 26 items having 4 domains. ${ }^{[8]}$

Due to this pandemic lockdown, it is been notice that people faced difficulty in transportation, overcrowded hospital with confirmed COVID cases, overworked staffs, lack of medical equipment \& lack of attention lead to drastic changes like lack of support person at the stage of labor and maternal separation from newborn in the suspected case of COVID status. Pregnancies are facing the Nosophobia \& are panic regarding the virus that may affect the developing fetus or infant which leads to poor health care and increased the risk of poor.

Human behavioral changes, such as fear, aversion to social gatherings, work and religious places and regular checkup leads to stressful events for childbearing women. They've to walk inside the dwelling place, not able to go outside to meet their families and meet new people leads to frustration and anxiety. Impacts of covid 19 on household income as many of the females mentioned financial crisis at peak level than usual which leads to economic disruption in different ways.

In compliance or following with other research studies conducted across the globe this study examined that the covid19 pandemics has subsequently affected the quality of life in child bearing women immediately after the pandemic period.

\section{METHODOLOGY}

Research design: Observational study
Study sample: 100 post-natal women aged between 18 to 39 years.

Sampling method: convenient sampling

Sample size: 100 subjects

Setting: Patients from OBG department and private hospitals in Bangalore.

\section{Inclusion criteria:}

1. Given birth during pandemic time.

2. Primigravida and Multigravida

3. Both LSCS and VD

\section{Exclusion criteria:}

1. Those who were not pregnant in pandemic time

2. High risk pregnancy

3. Those who didn't give birth during pandemic months

4. Unconscious patient

5. Severe illness

\section{PROCEDURE}

The survey was conducted on 100 women who delivered their babies throughout the time of covid 19 pandemic in Private Hospitals of OBG department in Bangalore.

A commonly accepted WHOQOFBREF scale was used in this study. It consists of 26-item instrument covering physical, psychological, social relationships and environmental health in 4 domains which was clearly explained to delivered women (18- to 39-year-old).

The domain of each item on a response scale is attain from 1 to $5 \&$ are converted to a 0-100 scale.

The purpose of the procedure and the survey was explained to each participant and consent were taken. Literate women filled their form by themselves whereas the illiterate answered their question through my support (researcher).

The basic demographic data and medical history (pregnancy related) of each individual were collected, and WHOQOLBREF scale has been employed to access impacts of covid 19 pandemic and quality of life in women immediately after childbirth. 
Revathi. S et.al. Impacts of COVID-19 pandemic and quality of life in women immediately after childbirth: observational study.

\section{RESULT}

Table-1 showing frequency distribution on age
\begin{tabular}{|l|l|l|}
\hline Age in years & F & \% \\
\hline $20-24$ & 31 & 31 \\
\hline $25-29$ & 37 & 37 \\
\hline $30-34$ & 24 & 24 \\
\hline $35-39$ & 8 & 8 \\
\hline Total & 100 & 100 \\
\hline
\end{tabular}

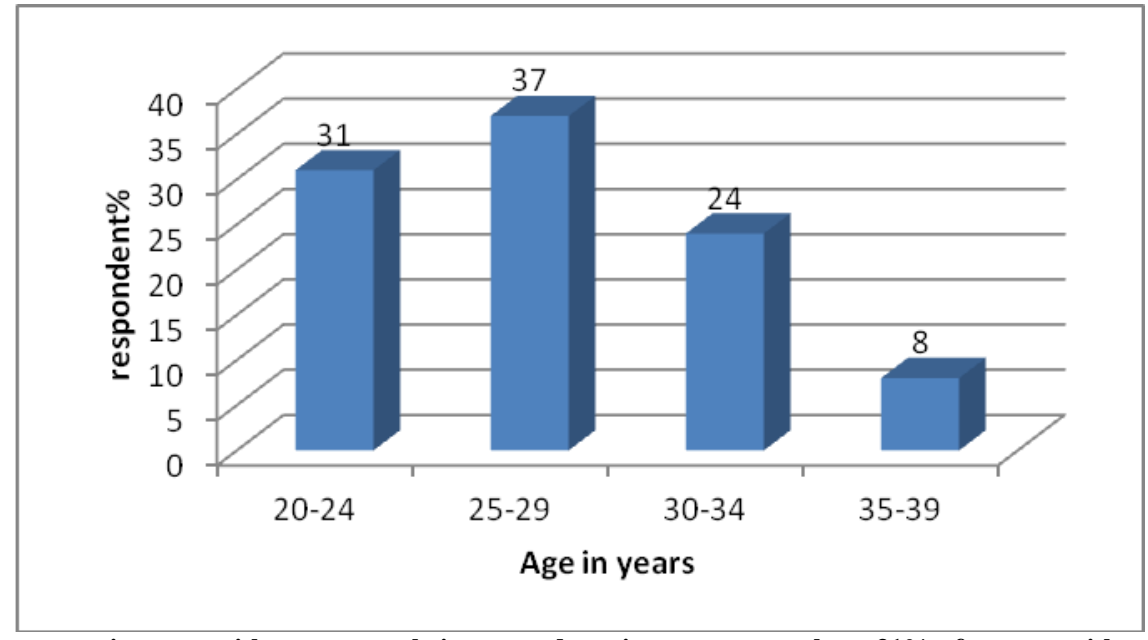

Fig- 01: graph shows age in years with respect to their respondents in percentage where $31 \%$ of women with age group of 20 -24, $37 \%$ of women with age group of $25-29,24 \%$ of $30-34$ age group and $8 \%$ of women with $35-39$ age groups have been participated

Table-2 showing domain wise mean and standard deviation

\begin{tabular}{|l|l|l|l|l|}
\hline Domain & Minimum & Maximum & Mean & Std. Deviation \\
\hline Domain1 & 31.00 & 94.00 & 55.2772 & 10.00012 \\
\hline Domain2 & 19.00 & 94.00 & 59.5644 & 13.29693 \\
\hline Domain3 & 44.00 & 94.00 & 67.5446 & 12.53357 \\
\hline Domain4 & 31.00 & 94.00 & 54.7624 & 14.22754 \\
\hline
\end{tabular}

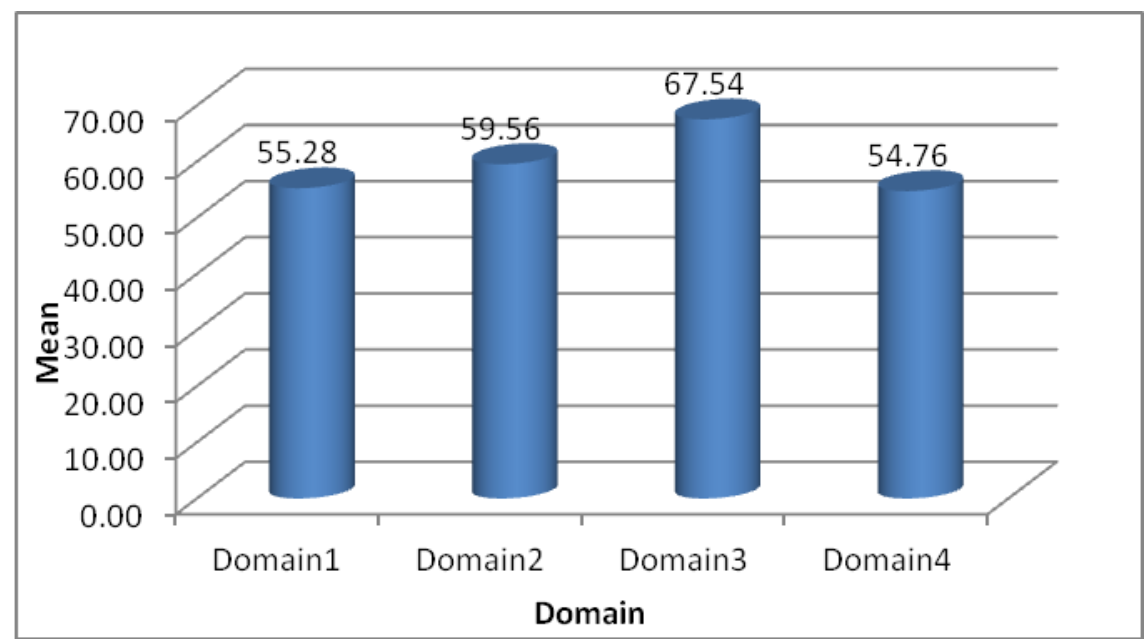

Fig- 02: graph showing mean of all four domains

Table-3: showing correlation between domains

\begin{tabular}{|l|l|l|l|}
\hline Correlation & r value & P value & Result \\
\hline Domain1_Domain2 & $.541^{* *}$ & .000 & $\mathrm{P}<.05 \mathrm{sig}$ \\
\hline Domain1_Domain3 & $.345^{* *}$ & .000 & $\mathrm{P}<.05 \mathrm{sig}$ \\
\hline Domain1_Domain4 & $.649^{* *}$ & .000 & $\mathrm{P}<.05 \mathrm{sig}$ \\
\hline Domain2_Domain3 & $.432^{* *}$ & .000 & $\mathrm{P}<.05 \mathrm{sig}$ \\
\hline Domain2_Domain4 & $.618^{* *}$ & .000 & $\mathrm{P}<.05 \mathrm{sig}$ \\
\hline Domain3_Domain4 & $.532^{* *}$ & .000 & $\mathrm{P}<.05 \mathrm{sig}$ \\
\hline
\end{tabular}

In table 3 the correlation between domains shows p<0.05 i.e., Significant. It revealed the correlation between domains where $r$ value and $p$ value are figured out and the result shows $\mathrm{p}<0.05$ that indicate the result is significant. 
Revathi. S et.al. Impacts of COVID-19 pandemic and quality of life in women immediately after childbirth: observational study.

Table-4 showing comparison between domain
\begin{tabular}{|l|l|l|l|l|}
\hline & Mean difference & t value & p value & Result \\
\hline Domain1_Domain2 & 4.28713 & -3.738 & .000 & $\mathrm{P}<.05 \mathrm{sig}$ \\
\hline Domain1_Domain3 & 12.26733 & -9.441 & .000 & $\mathrm{P}<.05 \mathrm{sig}$ \\
\hline Domain1_Domain4 & .51485 & .477 & .634 & $\mathrm{P}>.05 \mathrm{not}$ sig \\
\hline Domain2_Domain3 & 7.98020 & -5.820 & .000 & $\mathrm{P}<.05 \mathrm{sig}$ \\
\hline Domain2_Domain4 & 4.80198 & 4.003 & .000 & $\mathrm{P}<.05 \mathrm{sig}$ \\
\hline Domain3_Domain4 & 12.78218 & 9.854 & .000 & $\mathrm{P}<.05 \mathrm{sig}$ \\
\hline
\end{tabular}

Table-4 shows comparison allying all domains. Comparison had been done with respect to mean difference $t$ value and $\mathrm{p}$ value where all the comparison between the domains shows significant i.e., $\mathrm{p}<0.05$ except the comparison allying in 1and 4 domain i.e., $\mathrm{p}=0.634$.

The need of the study was to find out the impacts of covid19 pandemic on quality of life in women immediately after childbirth. Statistical analysis was performed using Karl Pearson coefficient for correlation between domains and Paired $t$ test for comparison of domains. The significance level was set to $\mathrm{p}<0.05$. There was a significant or notable impact of covid19 pandemic on quality of life in women immediately after childbirth.

\section{DISCUSSION}

The purpose of the survey is to find out the impact of covid19 pandemic on quality of life in women immediately after childbirth.

The study was conducted in Private Hospitals of OBG department.

100 women who delivered their babies in the time of covid 19 pandemic from aged between 18-39 years were assessed for quality of life. A commonly accepted WHOQOL- Brief Scale was performed on each post-natal woman. It consists of 26 questions which was clearly explained to each participant after the completion of consent form.

In our study the post-natal women affected by covid-19 pandemic had at terrific impact on quality of life. The complications of Covid 19 on post-natal women are preterm birth, neonatal morbidity \& maternal mortality, sleeping disorder, anxiety, depression\& continue to be significant public health problems for pregnancies and the child.

More than half of the women were more anxious regarding their health during and after pregnancy. Postnatal women (Multigravida) mentioned there was an adequate difference before and throughout the pandemic like low income, immigrant background, difficult in transportation, missing regular antenatal checkups and lack of attention lead to drastic changes in pregnancy throughout the time of labor.

Postnatal women admitted in the hospital were in fear of cross infection. Psychological stress events throughout this span were highly reported.

As stated in the questionnaire, the domain 1having mean of 55.28 which is shown in fig 2 graph, domain 2 having 59.56 mean, domain 3 having mean of 67.54 $\&$ lastly domain 4 having 54.76 mean.

Most of the delivered women mentioned psychological disturbances in their health and deficit of social harmony \& relationship between people throughout the time of covid19 pandemic. They were anxious regarding their infants \& the current situation which shows negative effect on their sleeping pattern and fear at peak level. Lack of physical activity \&unable-to be engaged in any social environment made them feel lonely.

The result of the survey shows that the delivered women throughout the pandemic had significant effect on quality of life.

Depending upon the result of this survey it is concluded that there was a significant impact of covid-19 pandemic on QOL in women immediately after childbirth because in all the test $p<0.05$. Except the comparison allying in $1 \& 4$ domain i.e., the 
Revathi. S et.al. Impacts of COVID-19 pandemic and quality of life in women immediately after childbirth: observational study.

delivered women felt safer in the home environment throughout the pandemic.

\section{Limitation}

The limitation of the current study was the pandemic because of which the data collection of the survey was bit difficult and the conversation with the patients as a result of fear of covid wasn't easy at all.

\section{Further Recommendation}

New research studies can be done in future in larger samples to look how the changed routines and the increased stress events after childbirth affect their bond between partner and children \& also the covid 19 possible long-term effects among infected \& non-infected delivered women.

\section{CONCLUSION}

Depending upon the result of the survey it is concluded that there was a significant impact of COVID-19 pandemic on quality of life in women immediately after childbirth because all the test shows $\mathrm{p}<0.05$.

\section{Acknowledgement: None}

\section{Conflict of Interest: None}

\section{Source of Funding: None}

\section{Ethical Approval: Approved}

\section{REFERENCES}

1. Gildner TE, Thayer ZM. Maternal and child health during the COVID-19 pandemic: Contributions in the field of human biology. American Journal of Human Biology. 2020 Jan 1.

2. Basu A, Kim HH, Basaldua R, Choi KW, Charron L, Kelsall N, Hernandez-Diaz S, Wyszynski DF, Koenen KC. A cross- national study of factors associated with women's perinatal mental health and wellbeing during the COVID-19 pandemic. PloS one. 2021 Apr 21;16(4):e0249780.

3. Mullins E, Evans D, Viner RM, O'Brien P, Morris E. Coronavirus in pregnancy and delivery: rapid review. Ultrasound in Obstetrics \& Gynecology. 2020 May; 55(5):586-92.

4. Alder J, Fink N, Bitzer J, Hösli I, Holzgreve W. Depression and anxiety during pregnancy: a risk factor for obstetric, fetal and neonatal outcome? A critical review of the literature. The Journal of Maternal-Fetal \& Neonatal Medicine. 2007 Jan 1;20(3):189-209

5. Kinsella MT, Monk C. Impact of maternal stress, depression \& anxiety on fetal neurobehavioral development. Clinical obstetrics and gynecology. 2009 Sep;52(3):425..

6. Davis-Floyd R, Gutschow K, Schwartz DA. Pregnancy, birth and the COVID-19 pandemic in the United States. Medical Anthropology. 2020 Jul 3;39(5):413-27.

7. Tran BX, Nguyen HT, Le HT, Latkin CA, Pham HQ, Vu LG, Le XT, Nguyen TT, Pham QT, Ta NT, Nguyen QT. Impact of COVID-19 on economic well-being and quality of life of the Vietnamese during the national social distancing. Frontiers in psychology. 2020:2289.

8. Van Esch L, Den Oudsten BL, De Vries J. The World Health Organization quality of life instrument-short form (WHOQOLBREF) in women with breast problems. International Journal of Clinical and Health Psychology. 2011;11(1):5-22.

How to cite this article: Revathi. S, Sukriti Rijal, Vijaya Krishna Kumar. Impacts of COVID-19 pandemic and quality of life in women immediately after childbirth: observational study. Int J Health Sci Res. 2022; 12(3):69-73. DOI: https://doi.org/10.52403/ijhsr.20220310 